\title{
Simulation of Monolithically Integrated Meta-Lens with Colloidal Quantum Dot Infrared Detectors for Enhanced Absorption
}

\author{
Yan Ning, Shuo Zhang, Yao Hu, Qun Hao * and Xin Tang * \\ Department of Optoelectronics, Beijing Institute of Technology, Beijing 100081, China; \\ ningyan516@163.com (Y.N.); 3120195342@bit.edu.cn (S.Z.); huy08@bit.edu.cn (Y.H.) \\ * Correspondence: qhao@bit.edu.cn (Q.H.); xintang@bit.edu.cn (X.T.)
}

\begin{abstract}
Colloidal quantum dots (CQDs) have been intensively investigated over the past decades in various fields for both light detection and emission applications due to their advantages like low cost, large-scale production, and tunable spectral absorption. However, current infrared CQD detectors still suffer from one common problem, which is the low absorption rate limited by CQD film thickness. Here, we report a simulation study of CQD infrared detectors with monolithically integrated meta-lenses as light concentrators. The design of the meta-lens for $4 \mu \mathrm{m}$ infrared was investigated and simulation results show that light intensity in the focused region is $\sim 20$ times higher. Full device stacks were also simulated, and results show that, with a meta-lens, high absorption of $80 \%$ can be achieved even when the electric area of the CQD detectors was decreased by a factor of 64. With higher absorption and a smaller detector area, the employment of meta-lenses as optical concentrators could possibly improve the detectivity by a factor of 32. Therefore, we believe that integration of CQD infrared detectors with meta-lenses could serve as a promising route towards high performance infrared optoelectronics.
\end{abstract}

Keywords: colloidal quantum dots; meta-lens; detectivity; optical concentrator

\section{Introduction}

With steady advancement, the maximum absorption wavelength of colloidal quantum dots (CQDs) has been extended from near-infrared to longer wavelength, providing a promising alternative to bulk infrared semiconductors like $\mathrm{HgCdTe}, \mathrm{InSb}$ and type-II superlattices [1,2]. Among all the colloidal nanomaterials, mercury telluride (HgTe) CQDs have so far demonstrated the highest spectral tunability from the short-wave infrared (SWIR) [3,4] and mid-wave infrared (MWIR) [5-9] to the long-wave infrared (LWIR) $[10,11]$ and terahertz (THz) region [12]. HgTe CQD-based photoconductors [7,8,13], photovoltaics [5,6,9], and phototransistors [14,15] have all been studied. Among them, HgTe CQD photovoltaic detectors have unique advantages like the absence of $1 / f$ noise [9] and fast operation speed [5]. SWIR detectors with detectivity up to $5 \times 10^{10}$ Jones at room temperature [16], MWIR detectors with background-limited infrared photodetection (BLIP) performance at cryogenic temperature [6], SWIR hyperspectral detectors with 64 channels [17], CQD-silicon dual-channel detectors [18], and SWIR/MWIR dual-band imagers with vertical junctions [19] have all been demonstrated in photovoltaic configuration. Despite all the exciting achievements, most infrared CQD detectors still suffer from one common problem, which is the low absorption rate limited by the film thickness of CQDs. CQD detectors are usually made by layer-by-layer drop-casting, and for an infrared HgTe CQD detector, the regular thickness is $\sim 400 \mathrm{~nm}$ and only $20 \%$ of incident light can be absorbed in the MWIR region [6]. Further increase of film thickness would lead to cracks and delamination due 
to mechanical stress from the drying and ligand exchange process [20]. Therefore, to improve the performance of infrared CQD photodetectors, one needs to find a strategy to enhance light absorption without increasing CQD film thickness.

For infrared detectors, the key figure of merit is detectivity, $D^{*}$, which can be expressed by

$$
D^{*}=\frac{\sqrt{A}}{I_{n}} \mathfrak{R}
$$

where $A$ is the light sensing area, $I_{n}$ is noise current, and $\mathfrak{R}$ is responsivity, which is related to absorption $\alpha$ by

$$
\mathfrak{R}=\frac{\alpha e}{h v} \eta
$$

where $e$ is elementary charge, $h$ is the Planck constant, $v$ is the frequency of photon, and $\eta$ is internal quantum efficiency. Therefore, the absorption of CQD film is related to $D^{*}$ by

$$
D^{*}=\frac{\sqrt{A}}{I_{n}} \frac{\alpha e}{h v} \eta
$$

Strategies of using metallic plasmonic nanostructures, resonant cavities, and photonic crystals have all been reported to boost CQD absorption up to 2-3 times [6,16]. Those structures are fabricated using regular lithographic processes [11] or multi-beam laser interference lithography [21,22], which may change the surface condition of CQDs and introduce noise source into CQD detectors. To optimize $D^{*}$, it is highly favorable to find a way to increase light absorption and decrease noise at the same time. The noise of a CQD photovoltaic detector mainly consists of Johnson noise, which scales with detector electric area $A_{e}$ by a factor of $A_{e}^{1 / 2}$ [18]. Employment of optical concentrators, therefore, seems to be a suitable method to address such a challenge. With light concentrators, the electric area of a detector can be decreased while maintaining the same responsivity by improving light absorption, leading to enhanced detectivity. Compared to three-dimensional (3D) microlenses or microspheres, meta-lenses can be fabricated in scalable ways and can provide functionalities like controlling the phase, amplitude, and polarization of the incident light by simply altering the shape and the size of each meta-unit [23,24]. $\mathrm{HgCdTe}$ and $\mathrm{GaSb}$ detectors with meta-lenses have been reported. However, such a concept has rarely been investigated for infrared CQD photodetectors.

Here, we report a simulation study of CQD infrared detectors with a monolithically integrated meta-lens as light concentrator. The target spectral range is MWIR $(3-5 \mu \mathrm{m})$. The design of the meta-lens in the center of MWIR $(4 \mu \mathrm{m})$ is discussed. The simulation results showed that light intensity in the focused region is 20 times higher than that of the incident light. More importantly, we find that the chromatic aberration of the meta-lens might unintentionally help the development of spectrally selective infrared detectors, which can be used to distinguish wavelength information of incident light. Besides simulation of the meta-lens, full device stacks were also simulated, and results showed that with a meta-lens, absorption of $80 \%$ can be achieved even when the electric area of the CQD detector was decreased by a factor of 64 . Therefore, we believe that integration of CQD infrared detectors with a meta-lens could serve as a promising route towards high performance infrared optoelectronics.

\section{Modeling and Simulation}

COMSOL Multiphysics is used to conduct the simulation in our study. To save computation resources, two-dimensional (2D) simulation is used as shown in Figure 1. The light is introduced from the "incident port" with controlled power of $1 \mathrm{~W}$. A listening port is placed on the top of the model. The left and right sides are set as "Scattering Boundary Condition" to avoid light reflection. The substrate material is chosen to be $\mathrm{Al}_{2} \mathrm{O}_{3}$ with a refractive index of $1.6+0 i$. The meta-lens units are made of silicon with a refractive index of $3.5+0 i$. For HgTe CQDs, the refractive index is $2.3+0.1 i[6]$. The rest of the model is set to be air with a refractive index of $1+0 i$. The absorption of CQDs is 
calculated by comparing the incident power and outlet power. The wavelength of incident light is swept from 1.5 to $5.0 \mu \mathrm{m}$ to calculate the spectral absorption. In out simulation, the diameter of the meta-lens is set to be $200 \mu \mathrm{m}$ to match with our commonly used size of detector in experimental tests. The phase profile and meta-lens design are generated by using open source software called MetaOptics (version v2) [25]. In our design, we use an unpolarized plane wave with uniform intensity distribution across the meta-lens region. To lower fabrication complexity, a pillar structure with periodicity of $2 \mu \mathrm{m}$ and height of $6 \mu \mathrm{m}$ was used. Complete phase shift of $0-2 \pi$ can be realized by changing the diameter from 0.6 to $1.7 \mu \mathrm{m}$. Further shrinking the size of the meta-lens (periodicity, diameter, and height) should still work as long as the light can still be focused onto the CQD detector.

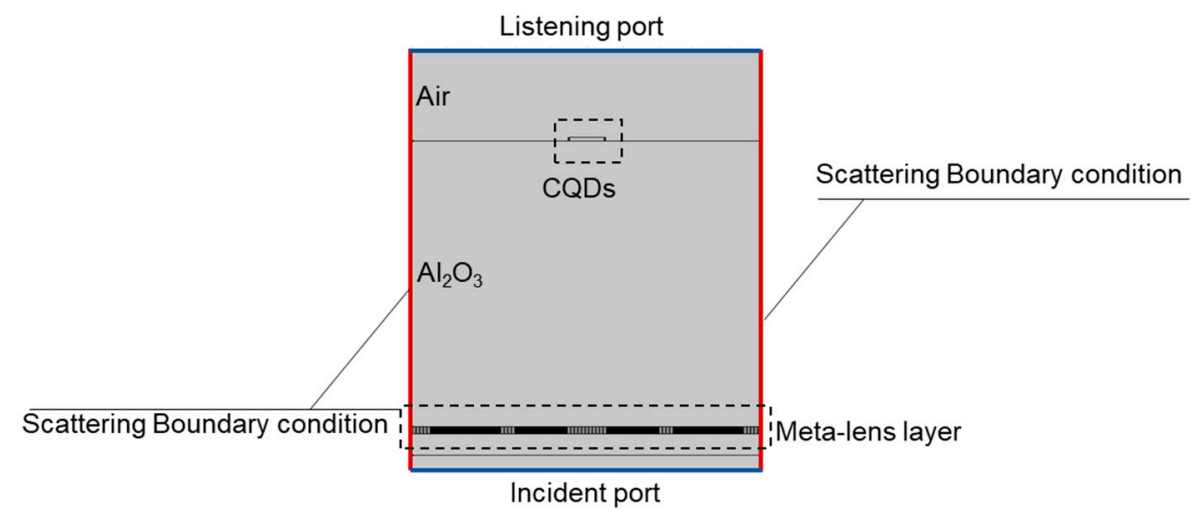

Figure 1. Simulation model of CQD detector integrated with a meta-lens.

\section{Results and Discussions}

The proposed design of CQD photodetectors with a monolithically integrated meta-lens is illustrated in Figure 2a. Pillar structures made of silicon were selected as the material to construct the meta-lens unit. The incident light illuminated on the meta-lens can be concentrated and focused into the CQD detector. As shown in the cross-sectional illustration in Figure 2b, the electric area of the CQD detector is much smaller than the optical area of the meta-lens. To design a functional meta-lens, the first step is to calculate the required phase profile based on the lens equation

$$
\phi(x, y)=\frac{2 \pi}{\lambda} n_{m}\left(f-\sqrt{x^{2}+y^{2}+f^{2}}\right)
$$

where $\lambda$ is the targeted wavelength, $n_{m}$ is the index of refraction of the medium, and $f$ is the focal length. For a regular imaging lens, the phase profile for $\lambda=4 \mu \mathrm{m}$ and $f=300 \mu \mathrm{m}$ is shown in Figure $2 \mathrm{c}$ (black curve). For a meta-lens, the continuous phase profile modulation is achieved by periodically changing the effective index of refraction of the medium, which can be done by tuning the height and diameter of the silicon pillar in the meta-lens unit. The red curve in Figure $2 \mathrm{c}$ shows the required phase profile of a meta-lens, based on which the meta-lens design was generated using open source software called MetaOptics. In our design, a pillar structure with periodicity of $2 \mu \mathrm{m}$ and height of $6 \mu \mathrm{m}$ was used. By changing the diameter from 0.6 to $1.7 \mu \mathrm{m}$, a phase shift of $0-2 \pi$ was covered. Figure $2 \mathrm{~d}$ shows the 2D phase profile mapping and structure design of a meta-lens. 

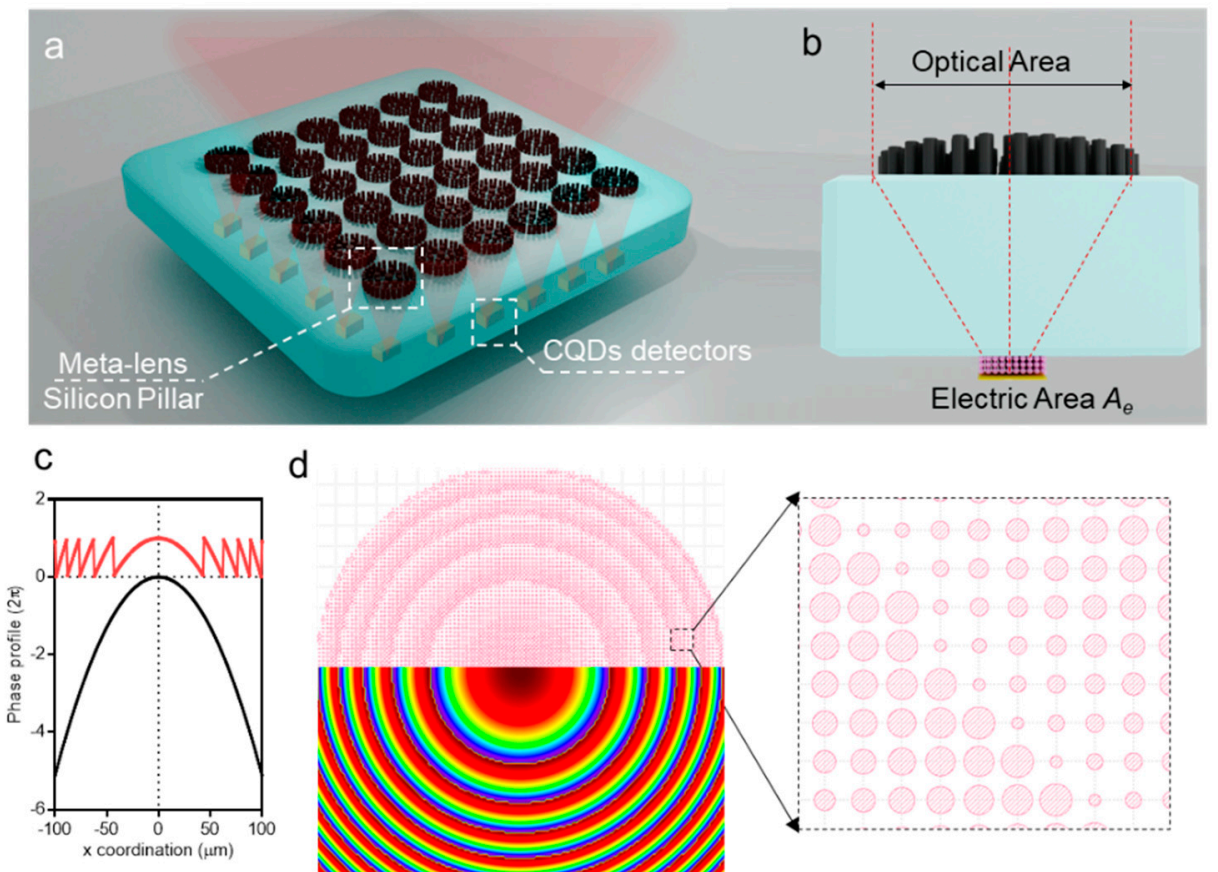

Figure 2. (a) Illustration of meta-lens integrated with CQD detectors. Incident light is collected and focused into CQD detectors with a small electric area. (b) Cross-sectional illustration of the meta-lens integrated with the CQD detector. Light illuminated onto the optical area is focused to the CQD detector with electric area $A_{e}$. (c) Phase profile of a regular lens (black curve) and meta-lens (red). (d) Design of meta-lens using pillar structures. The diameter and focal length are 200 and $200 \mu \mathrm{m}$, respectively. Inset is a representative $2 \mathrm{D}$ phase mapping used to generate the meta-lens design.

To verify the optical function of the meta-lens design, the light intensity distribution after passing through the meta-lens is simulated. Various values of numerical aperture (NA) were used and the results showed that the incident light could be successfully concentrated and focused into a smaller spot and that the focal length could be precisely tuned by changing the geometrical parameters of the meta-lens (Figure 3a). For a meta-lens with diameter of $200 \mu \mathrm{m}$, the diameter of the focused region is around 10-20 $\mu \mathrm{m}$, depending on the NA (Figure 3b). Overall, the enhancement ratio of light intensity in the focused region is $\sim 20$, which is similar to previously reported experimental values [26].

Besides simulation of the meta-lens with different NAs, chromatic aberration was also investigated. For the meta-lens with a NA of 0.43 and targeted wavelength of $4 \mu \mathrm{m}$, we calculated the intensity distribution for incident light with different wavelengths. Chromatic aberration was clearly found, as shown in Figure $4 \mathrm{a}$. As the wavelength deviates from the targeted center wavelength of $4 \mu \mathrm{m}$, the focal spot obviously shifts, moving the focused light away from the designed position where the CQD detector would be located. Chromatic aberration limits the operation spectral ranges of the meta-lens and is hard to correct. However, such properties could be potentially used to develop infrared detectors with spectral selectivity (Figure $4 \mathrm{~b}$ ). Spectral selectivity presents in all three meta-lenses $(2.5,4.0$, and $4.8 \mu \mathrm{m})$. Away from the targeted wavelength, the enhancement ratio decreases rapidly. The simulation results clearly demonstrate the versatility and flexibility of meta-lenses. 

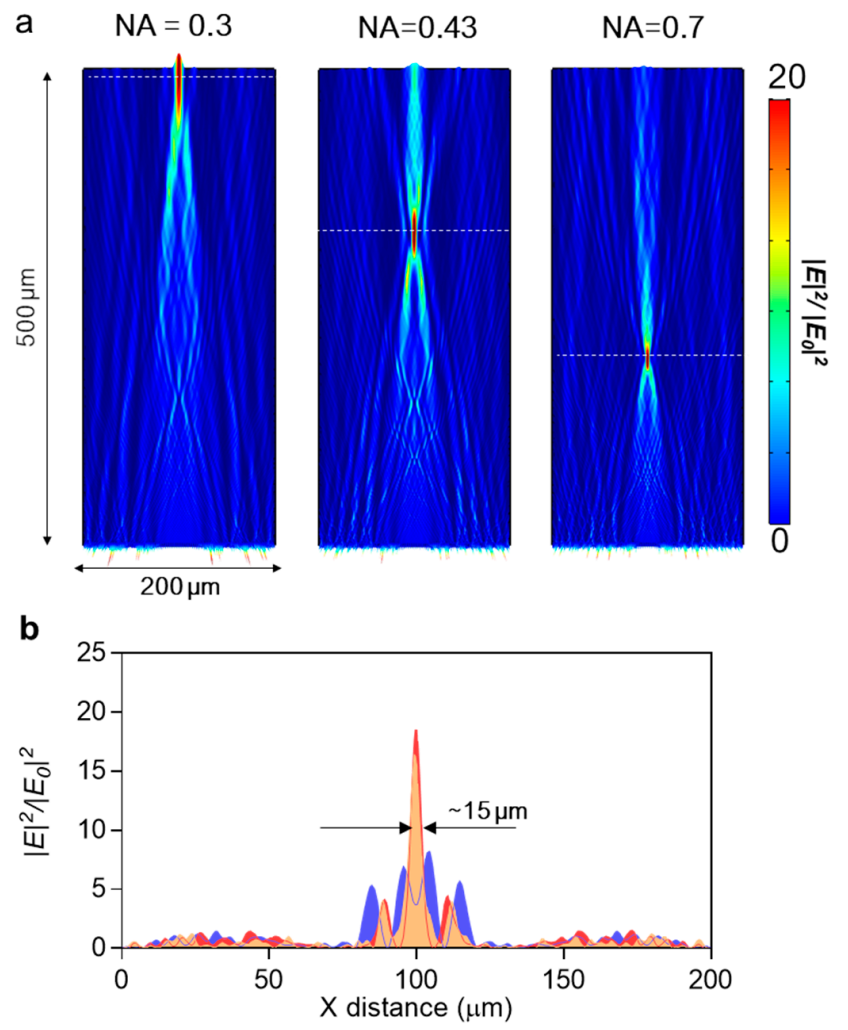

Figure 3. (a) COMSOL simulation of light intensity distribution with various numerical apertures (NAs). (b) Enhancement ratio profile across the focused area (white dashed line in Figure 2a).

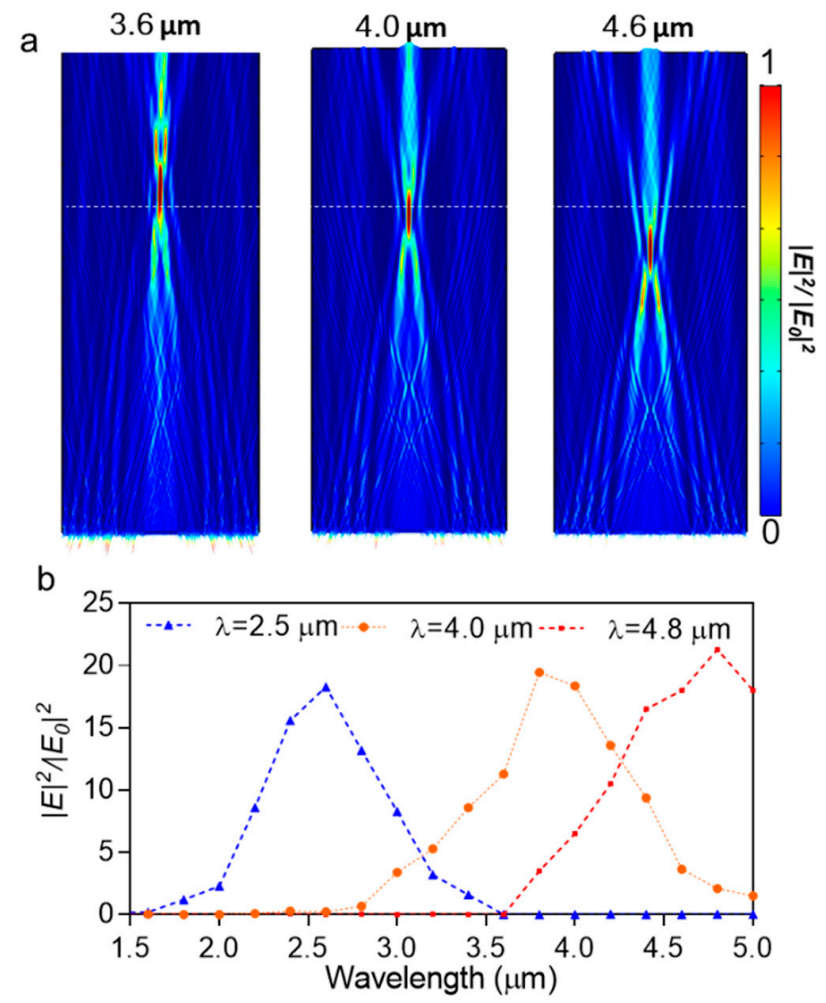

Figure 4. (a) Simulated light intensity distribution of a meta-lens with NA of 0.43 with light at different wavelength. (b) Spectral enhancement factors across the focused area for meta-lens designed for 2.5, 4.0, and $4.8 \mu \mathrm{m}$ (dashed line in Figure 3a). 
Finally, we simulated the full CQD device stacks with a meta-lens using COMOSL. The simulation model is shown in Figure 5a. The incident light is first focused by the meta-lens and then absorbed by the CQD layers. The diameter of the meta-lens is $200 \mu \mathrm{m}$ and the width of the CQD detector is swept from 5 to $30 \mu \mathrm{m}$ with the same thickness of $400 \mathrm{~nm}$. As shown in the simulated light intensity distribution mapping, most of the incident light can be focused onto the CQD detectors by tuning the geometrical parameters of the meta-lens (Figure 5b). With the width of the CQD detectors increasing from 5 to $30 \mu \mathrm{m}$, the absorption gradually increases from $10 \%$ to $80 \%$ and saturates at a width of $25 \mu \mathrm{m}$ (Figure 5c). Compared to the reference detector with a width of $200 \mu \mathrm{m}$ without a meta-lens, it is impressive that 4 times more light can be absorbed by a smaller detector at the targeted wavelength of $\sim 4 \mu \mathrm{m}$. Based on Equation (3) and previous discussion, higher absorption leads to higher responsivity $\mathfrak{R}(\times 4)$ and smaller detector results in smaller noise current $(\times 0.125)$. Therefore, the employment of meta-lenses as optical concentrators could improve the detectivity by a factor of 32 . Previous experimental studies of $\mathrm{HgCdTe}$ with meta-lenses reported an enhancement ratio of $~ 5.5$, demonstrating that the concept of integration of meta-lenses with infrared detectors should work [26]. The current study mainly focuses on simulation and theoretical calculation, and experimental tests would be conducted to verify such an enhancement factor in the future.
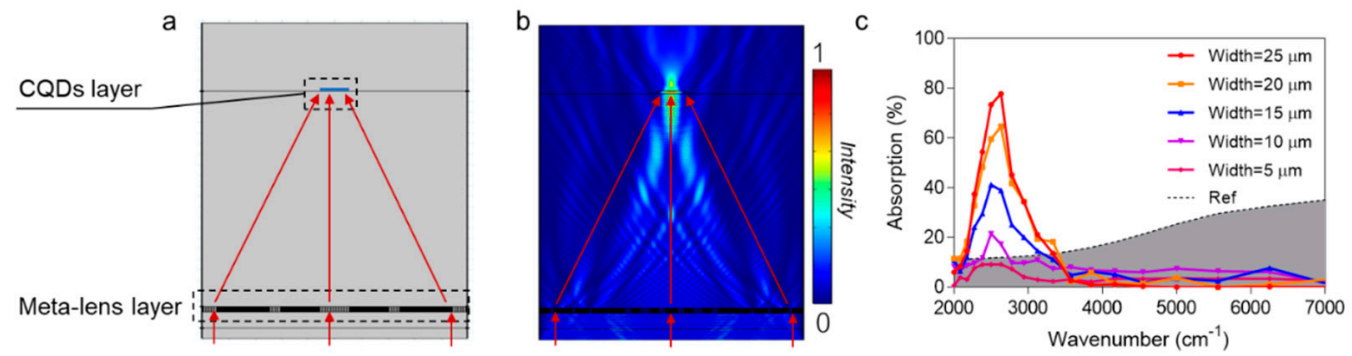

Figure 5. (a) Simulation model used to calculate the absorption of CQD detector with meta-lens. (b) Simulated light intensity distribution of light passing through meta-lens. (c) Simulated absorption of CQD detector with width of 5 to $25 \mu \mathrm{m}$ with meta-lens and simulated absorption from a reference detector with a width of $200 \mu \mathrm{m}$ (no meta-lens).

\section{Conclusions}

In conclusion, our simulation results show that integration of meta-lenses with CQD detectors is a promising way to improve detector detectivity by increasing absorption and decreasing noise at the same time. Full device stack simulation shows that, with meta-lenses, a high absorption of $80 \%$ can be achieved even when the electric area of the CQD detectors is decreased by a factor of 64 . With meta-lenses as optical concentrators, the detectivity can be improved by a factor of 32. By drop-casting HgTe CQDs on a commercial read-out integrated circuit, a previous study demonstrated that a MWIR thermal camera with a noise equivalent differential temperature (NEDT) of $102 \mathrm{mK}$ [27] showed a simple fabrication route to low-cost thermal cameras with CQDs. Therefore, we believe that integration of CQD infrared detectors with meta-lenses could serve as a promising route towards both high performance single-element detectors and focal plane arrays.

Author Contributions: Conceptualization, X.T.; Methodology, Y.N., S.Z., and Y.H.; Supervision, X.T. and Q.H.; All authors have read and agreed to the published version of the manuscript.

Funding: This work was supported by the National Science Foundation of China (NSFC) (62035004).

Conflicts of Interest: The authors declare no conflict of interest.

\section{References}

1. Rogalski, A. History of infrared detectors. Opto-Electron. Rev. 2012, 20, 279-308. [CrossRef]

2. Rogalski, A. Toward third generation HgCdTe infrared detectors. J. Alloys Compd. 2004, 371, 53-57. [CrossRef] 
3. Böberl, M.; Kovalenko, M.V.; Gamerith, S.; List, E.J.W.; Heiss, W. Inkjet-printed nanocrystal photodetectors operating up to $3 \mu \mathrm{m}$ wavelengths. Adv. Mater. 2007, 19, 3574-3578. [CrossRef]

4. Jagtap, A.; Goubet, N.; Livache, C.; Chu, A.; Martinez, B.; Gréboval, C.; Qu, J.; Dandeu, E.; Becerra, L.; Witkowski, N.; et al. Short wave infrared devices based on HgTe nanocrystals with air stable performances. J. Phys. Chem. C 2018, 122, 14979-14985. [CrossRef]

5. Ackerman, M.M.; Tang, X.; Guyot-Sionnest, P. Fast and sensitive colloidal quantum dot mid-wave infrared photodetectors. ACS Nano 2018, 12, 7264-7271. [CrossRef] [PubMed]

6. Tang, X.; Ackerman, M.M.; Guyot-Sionnest, P. Thermal imaging with plasmon resonance enhanced HgTe colloidal quantum dot photovoltaic devices. ACS Nano 2018, 12, 7362-7370. [CrossRef] [PubMed]

7. Keuleyan, S.; Lhuillier, E.; Guyot-Sionnest, P. Synthesis of colloidal HgTe quantum dots for narrow mid-IR emission and detection. J. Am. Chem. Soc. 2011, 133, 16422-16424. [CrossRef] [PubMed]

8. Keuleyan, S.; Lhuillier, E.; Brajuskovic, V.; Guyot-Sionnest, P. Mid-infrared HgTe colloidal quantum dot photodetectors. Nat. Photonics 2011, 5, 489-493. [CrossRef]

9. Guyot-Sionnest, P.; Roberts, J.A. Background limited mid-infrared photodetection with photovoltaic HgTe colloidal quantum dots. Appl. Phys. Lett. 2015, 107, 253104. [CrossRef]

10. Keuleyan, S.E.; Guyot-Sionnest, P.; Delerue, C.; Allan, G. Mercury telluride colloidal quantum dots: Electronic structure, size-dependent spectra, and photocurrent detection up to $12 \mu \mathrm{m}$. ACS Nano 2014, 8, 8676-8682. [CrossRef]

11. Tang, X.; Wu, G.F.; Lai, K.W.C. Plasmon resonance enhanced colloidal HgSe quantum dot filterless narrowband photodetectors for mid-wave infrared. J. Mater. Chem. C 2017, 5, 362-369. [CrossRef]

12. Goubet, N.; Jagtap, A.; Livache, C.; Martinez, B.; Portalès, H.; Xu, X.Z.; Lobo, R.P.S.M.; Dubertret, B.; Lhuillier, E. Terahertz HgTe nanocrystals: Beyond confinement. J. Am. Chem. Soc. 2018, 140, 5033-5036. [CrossRef] [PubMed]

13. Chen, M.; Yu, H.; Kershaw, S.V.; Xu, H.; Gupta, S.; Hetsch, F.; Rogach, A.L.; Zhao, N. Fast, Air-stable infrared photodetectors based on spray-deposited aqueous HgTe quantum dots. Adv. Funct. Mater. 2014, 24, 53-59. [CrossRef]

14. Lhuillier, E.; Robin, A.; Ithurria, S.; Aubin, H.; Dubertret, B. Electrolyte-gated colloidal nanoplatelets-based phototransistor and its use for bicolor detection. Nano Lett. 2014, 14, 2715-2719. [CrossRef] [PubMed]

15. Huo, N.; Gupta, S.; Konstantatos, G. $\mathrm{MoS}_{2}-\mathrm{HgTe}$ quantum dot hybrid photodetectors beyond $2 \mu \mathrm{m}$. Adv. Mater. 2017, 29, 1606576. [CrossRef]

16. Tang, X.; Ackerman, M.M.; Shen, G.; Guyot-Sionnest, P. Towards infrared electronic eyes: Flexible colloidal quantum dot photovoltaic detectors enhanced by resonant cavity. Small 2019, 15, 1804920. [CrossRef]

17. Tang, X.; Ackerman, M.M.; Guyot-Sionnest, P. Acquisition of hyperspectral data with colloidal quantum dots. Laser Photon. Rev. 2019, 13, 1900165. [CrossRef]

18. Tang, X.; Chen, M.; Kamath, A.; Ackerman, M.M.; Guyot-Sionnest, P. Colloidal quantum-dots/graphene/silicon dual-channel detection of visible light and short-wave infrared. ACS Photonics 2020, 7, 1117-1121. [CrossRef]

19. Tang, X.; Ackerman, M.M.; Chen, M.; Guyot-Sionnest, P. Dual-band infrared imaging using stacked colloidal quantum dot photodiodes. Nat. Photonics 2019, 13, 277-282. [CrossRef]

20. Tang, X.; Chen, M.; Ackerman, M.M.; Melnychuk, C.; Guyot-Sionnest, P. Direct imprinting of quasi-3D nanophotonic structures into colloidal quantum-dot devices. Adv. Mater. 2020, 32, 1906590. [CrossRef]

21. Gedvilas, M.; Voisiat, B.; Indrišiūnas, S.; Račiukaitis, G.; Veiko, V.; Zakoldaev, R.; Sinev, D.; Shakhno, E. Thermo-chemical microstructuring of thin metal films using multi-beam interference by short (nano- \& picosecond) laser pulses. Thin Solid Films 2017, 634, 134-140. [CrossRef]

22. Zhang, Z.; Dong, L.; Ding, Y.; Li, L.; Weng, Z.; Wang, Z. Micro and nano dual-scale structures fabricated by amplitude modulation in multi-beam laser interference lithography. Opt. Express 2017, 25, 29135. [CrossRef]

23. Zou, X.; Zheng, G.; Yuan, Q.; Zang, W.; Chen, R.; Li, T.; Li, L.; Wang, S.; Wang, Z.; Zhu, S. Imaging based on metalenses. PhotoniX 2020, 1, 1-24. [CrossRef]

24. Huang, T.Y.; Grote, R.R.; Mann, S.A.; Hopper, D.A.; Exarhos, A.L.; Lopez, G.G.; Kaighn, G.R.; Garnett, E.C.; Bassett, L.C. A monolithic immersion metalens for imaging solid-state quantum emitters. Nat. Commun. 2019, 10, 1-8. [CrossRef] [PubMed]

25. Dharmavarapu, R.; Hock Ng, S.; Eftekhari, F.; Juodkazis, S.; Bhattacharya, S. MetaOptics: Opensource software for designing metasurface optical element GDSII layouts. Opt. Express 2020, 28, 3505. [CrossRef] [PubMed] 
26. Li, F.; Deng, J.; Zhou, J.; Chu, Z.; Yu, Y.; Dai, X.; Guo, H.; Chen, L.; Guo, S.; Lan, M.; et al. HgCdTe mid-Infrared photo response enhanced by monolithically integrated meta-lenses. Sci. Rep. 2020, 10, 1-10. [CrossRef] [PubMed]

27. Buurma, C.; Pimpinella, R.E.; Ciani, A.J.; Feldman, J.S.; Grein, C.H.; Guyot-Sionnest, P. MWIR imaging with low cost colloidal quantum dot films. In Proceedings of the Optical Sensing, Imaging, and Photon Counting: Nanostructured Devices and Applications, San Diego, CA, USA, 8 December 2016; Razeghi, M., Temple, D.S., Brown, G.J., Eds.; International Society for Optics and Photonics: Bellingham, WA, USA, 2016; Volume 9933, p. 993303.

Publisher's Note: MDPI stays neutral with regard to jurisdictional claims in published maps and institutional affiliations.

(C) 2020 by the authors. Licensee MDPI, Basel, Switzerland. This article is an open access article distributed under the terms and conditions of the Creative Commons Attribution (CC BY) license (http://creativecommons.org/licenses/by/4.0/). 